\title{
Performance Enhancement of Telemedicine Network by Free Space Optics Links Provisioning
}

\author{
M. SiRAJ ${ }^{a, *}$, M. SHOAIB ${ }^{b}$ AND I. MEMON ${ }^{c}$ \\ ${ }^{a}$ College of Engineering, King Saud University, Riyadh, Saudi Arabia \\ ${ }^{b}$ PSATRI, College of Engineering, King Saud University, Riyadh, Saudi Arabia \\ ${ }^{c}$ Department of Computer Science, Muslim Youth University, Islamabad, Pakistan
}

\begin{abstract}
Health care has benefited immensely with the implementation of fast moving information technology. It is a challenging assignment for the researchers to implement the present day technologies such as wireless mesh networks. Wireless mesh networks are deployed to guarantee a reliable and stable network infrastructure backbone to telemedicine systems. They are preferred to other wireless networks, as the solution which they provide is robust for broadband services. They are robust because if routing is not possible due to some mesh nodes failure, routing can be done by the other mesh nodes present. Wireless mesh network cannot only spread coverage but also save both cabling cost and human resource. However, their performance is degraded due to interference and varying load. Interference is a critical issue in wireless mesh networks. The interference occurs because of the congested unlicensed radio frequency spectrum. This problem can be addressed by applying free space optics technology for transmission instead of radio frequency technology in the unlicensed spectrum. Network capacity issue is addressed by provisioning free space optics links to generate the much needed throughput and to minimize interference. Telemedicine network is a network, which requires a high capacity bandwidth to cater for a large number of remote patients. It is observed that free space optics links provisioning results in a significant performance enhancement for the telemedicine network.
\end{abstract}

DOI: 10.12693/APhysPolA.131.43

PACS/topics: 84.40.Ua

\section{Introduction}

Wireless mesh networks (WMNs) [1, 2] is a reliable novel technology, for providing broadband connectivity to the internet to the end users. They are self-healing, self-organizing, and self-forming. As they are easy to deploy and configure they have become immensely popular. They are reliable and economical. They are simple to be deployed, wherever and whenever they are required. Internet accessibility is easy for the end users as they can simply connect to any of the WMN routers. WMNs are being used as next generation networks. There are two elements of WMNs which are mesh clients and mesh router namely. Many present day applications like teleconferencing, video on demand, IP telephony, multimedia messaging and in telemedicine system are being implemented on WMN as it provides a high capacity and low implementation cost. After having such a stable infrastructure as a backbone, WMNs can assist in addressing many critical medical problems. Simultaneous wireless transmissions in WMNs causes interference [3, 4] which in turn reduces WMNs capacity. There are two major types of interference affecting multi-hop wireless networks throughput, which are intra-flow interference and interflow interference, respectively. Intra-flow interference is the interference arising on the same routing path between adjacent nodes. Inter-flow interference is the interference which arises from nodes from neighboring paths.

*corresponding author; e-mail: siraj@ksu.edu.sa
Telemedicine system is a present day technology, which provides specialized medical services to patients remotely. It is the involvement of present day technology, involving bidirectional interactive multimedia communication. It makes use of telemetry and computers for delivering state of art health services to patients remotely. It provides a facility to have a bidirectional interactive communication between physicians and specialists with the patients who are at a remote distance from each other so that the patient's health is maintained.

In Ref. [5], a telemedicine system was designed in Brazil, where its Teaching Hospitals and University were connected to national education and research network over 1 Gbps bandwidth. At the moment through this system a telemedicine system network consisting of 48 Universities and Teaching Hospitals operate. WMNs based telemedicine system $[6,7]$ are used in the industry. For providing a high capacity bandwidth to the telemedicine network, WMNs interference, which is a critical concern, has to be minimized.

Free space optical (FSO) communications are being considered due to a high bandwidth demand. Weather conditions play an important part on the functionality of FSO links. Performance of FSO links degrade with rain, scintillations, smoke, and fog.

\section{Related work}

Researchers have done many studies regarding RF/FSO links based networks on specific applications. An upper bound on the capacity of each node was deduced [8]. They incorporated FSO trans receivers and proved that upper bound capacity can be increased by 
a hybrid (RF/FSO) routing scheme. Hybrid RF/FSO mesh networks were investigated for node availability and reliability [9]. In [10] FSO links were introduced to minimize RF link interference with the smallest number of FSO links used. The network was optimized while maintaining a certain throughput. Many researchers have proposed/implemented various designs of hybrid networks [11-14].

Besides the hybrid mesh networks, researchers have used WMNs for its superior bandwidth. In Ref. [15] capacity of the network was improved through the exploitation of all optical multicast in semiconductor ring laser.

For future communication, FSO links are considered very important due to their high data rate. They are being considered now for space and terrestrial applications [16]. Unmanned aerial vehicles (UAVs), satellites, high altitude platforms, deep space probes are some of the FSO probable applications. They are also being considered for civilian and military applications. Their implementation is fairly easy. As a result, they are normally based on direct detection and intensity modulation. To achieve higher data rates, WDM based FSO systems are being deployed [17]. In this work, for the telemedicine network, WDM based FSO system with a data rate of $1.25 \mathrm{Gbps}$ is considered [18].

\section{Proposed model and problem formulation}

Figure 1 shows a detailed block diagram of telemedicine network model utilizing RF and FSO at the specialized hospital premises, which has been built on WMN

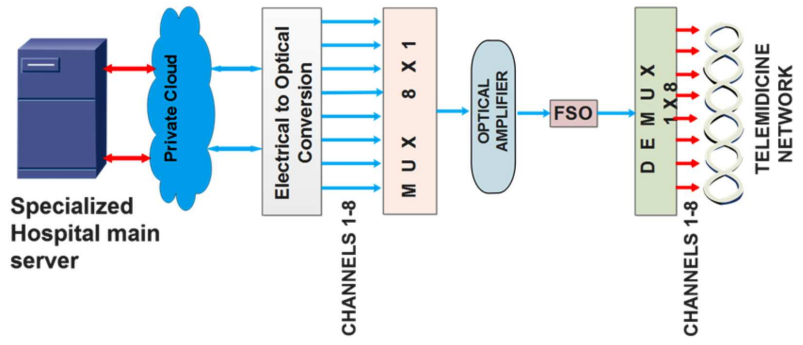

Fig. 1. Block diagram of telemedicine network over FSO links.

Figure 2 presents a conceptual model of our telemedicine network model (TNM) over FSO links.

The specialized hospital main server is connected to the network via a private cloud. Wireless local area network (WLANs), wide area network (WAN) and wired infrastructure constitute the main parts. The WLAN is connected to the internet through the wired infrastructure. Biosensors (mobile and stationary), radio frequency identification (RFID), personal digital assistant (PDA), wireless access pointers, etc. are some of the components of the WLANs networks.

The network is modeled as a directed graph. We epitomize a WMN with $n$ number of nodes scattered randomly by a directed graph. $G=(N, E)$, where $E$ is the set

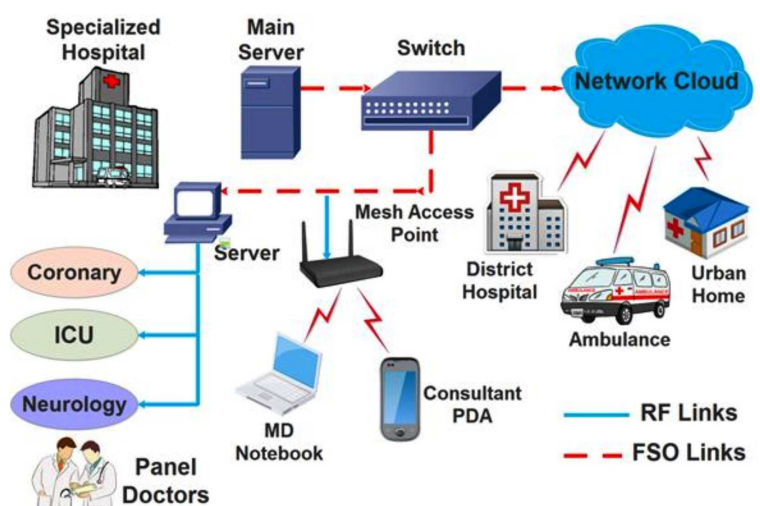

Fig. 2. A conceptual model of specialized hospital network with hybrid RF/ FSO mesh network.

of directional edges, representing radio frequency links and $N$ is vertices set signifying WMN nodes. Let the distance between $j$ and $k$ nodes be $a_{i j}$. All the nodes have a transmitter whose interference and transmission range are $b_{i}$ and $b_{t}$. A link occurs between node $I$ and node $j$ if

$$
a_{i j} \leq b_{i j} \text { and } i \neq j \text {. }
$$

Thus the directed edge $\{i, j\} \in E$ represents the link between $i$ and $j$ nodes. A connectivity matrix $T$ can be built for this directed graph $G(N, E)$. This connectivity matrix is based depends on the connection among the links and vertices. A directly connected can be represented by 1 whereas 0 signify the absence of connectivity. A node $i$ successfully transmits to node $j$, provided the conditions as represented by Eqs. (1) and (2) are met:

$a_{i j} \leq b_{t}$,

$a_{k j} \leq b_{i k}$, if $n_{k}$ is the neighbor node.

Similarly, an interference graph $G_{i}$ can be built. $G_{i}=$ $\left(N_{i}, E_{i}\right)$ represents the interference graph. A directed edge between node $j$ and node $k$ occurs provided:

a) presence of a strong signal from node $j$ that can disturb $k$,

b) $k$ is not able to distinguish it.

So if $\{j, k\}$ and $\{l, m\}$ communication links are present and the links are unable to transmit, it signifies the existence of an edge between them. Therefore, for interference graph $G_{i}$, edge can be constructed between $\{j, k\}$ and $\{l, m\}$. The condition is based on either $a_{j m} \leq b_{j}$ or $a_{l k} \leq b_{l}$.

From the vertex set $E_{i}$ and $G_{i}$, the interference graph of an interference vector can be constructed from any link with the rest of the links. If a link exists among links $\{\mathrm{k}$, $\mathrm{l}\}$ and $\{\mathrm{i}, \mathrm{j}\}$ then $I\{\mathrm{k}, \mathrm{l}\}\{\mathrm{i}, \mathrm{j}\}=1$ else 0 . Correspondingly for the entire links, interference vector can be calculated and an interference constraint matrix $|F| \times|F|$ can be built.

If a node $j$ wants to communicate to node $i$ on channel $y$, the nodes $j$ and $i$ must neither be transmitting nor 
receiving data on channel $y$. Moreover, node $i$ must not exist in node $k$ interference range on channel $y$. Channel $y$ status is given by the following equation:

$$
P_{i j}^{(y)}=\left\{\begin{array}{l}
1 \\
0
\end{array}\right\} .
$$

In our case we have taken two channels and rest of the frequency links have been assigned FSO link. It is a wellknown fact that FSO links communicate point to point without causing interference to the other RF links of the network.

\section{Simulation and performance results}

To evaluate the performance, network topology as shown in Fig. 3 was used. The mesh routers were arranged randomly in a $4 \times 4$ grid. The mesh routers had equal transmission powers. Each node was equidistant from its neighbor. Interference range was also assumed to be same for every node. RF and FSO links are used for communicating between nodes.

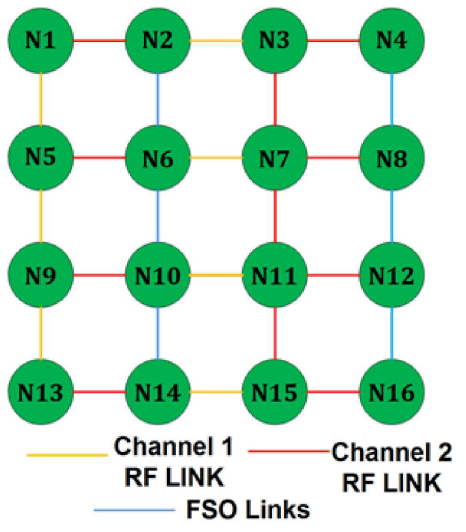

N1,N2....N16 MESH NODES

Fig. 3. A $4 \times 4$ mesh network with RF/FSO links.

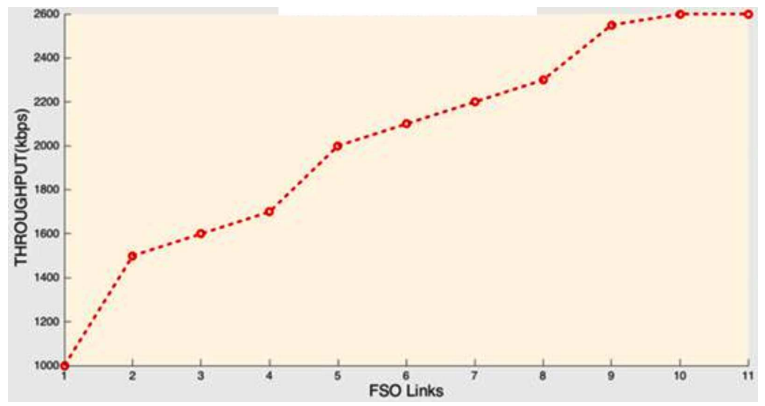

Fig. 4. Throughput for a 16 node network with increasing number of FSO links.

This is solved by simple linear programming (SLP) which is done in Visual Basic. Figure 4 shows the throughput with various numbers of FSO links. It is observed that there is a significant increase in throughput when RF links are replaced by FSO links. However, after a certain stage, increasing FSO links did not yield significant throughput. This can be due to the presence of external interfering agents and the placement of FSO links between the nodes. A suitable placement of FSO links will certainly yield a rich throughput.

\section{Conclusion}

In this work, we have proposed the use of FSO links along with RF links in WMN based telemedicine network. It is observed from the simulation results that performance of telemedicine network is enhanced through FSO links provisioning. Through the use of FSO links, the interference among WMN nodes is mitigated. By the suitable placement of FSO links, in place of interfering RF links, the throughput can be further enhanced. Since we have done the simulation for a $4 \times 4$ mesh scenario, it will be interesting to see the effects of RF and FSO links on larger mesh networks.

\section{References}

[1] M. Siraj, K.A. Bakar, Int. J. Phys. 6, 3189 (2011).

[2] M. Siraj, World Appl. Sci. J. 30, 870 (2014).

[3] M. Siraj, K.A. Bakar, Int. J. Phys. Sci. 7, 456 (2012).

[4] M. Siraj, Int. Inform. Inst. (Tokyo) 16, 7611 (2013).

[5] W. Coury, L.A. Messina, J.L.R. Filho, N. Simőes, in: Proc. 6th World Congress on Services, Miami (FL) 2010, p. 287.

[6] M. Siraj, K.A. Bakar, J. Comp. Sci. 8, 1263 (2012).

[7] M. Siraj, K.A. Bakar, World Appl. Sci. J. 18, 1271 (2012).

[8] D. Wang, A. Abouzeid, in: Proc. Information Theory and Applications Workshop, La Jolla (CA) 2007, p. 3.

[9] H. Moradi, M. Falahpour, H. Reafi, P. LoPresti, M. Atiquzzaman, in: Proc. INFOCOM IEEE Conf. on Computer Communications Workshops, San Diego (CA) 2010, p. 1.

[10] V. Rajakumar, M. Smadi, S. Ghosh, T. Todd, S. Hranilovic, J. Light Wave Technol. 26, 1735 (2008).

[11] A. Kashyap, M. Shayman, Proc. IEEE Int. Conf. on Communications 5, 3427 (2005).

[12] Jinlong Zhang, Proc. IEEE Int. Conf. on Communications 4, 2142 (2002).

[13] P.T. Dat, A. Bekkali, K. Kazaura, K. Wakamori, M. Matsumoto, J. Light Wave Technol. 28, 2258 (2010).

[14] T. Plank, E. Leitgeb, P. Pezzei, Z. Ghassemlooy, in: Proc. 17th Europ. Conf. on Networks and Optical Communications, Vilanova i la Geltru 2012, p. 1.

[15] M. Siraj, M.I. Memon, M. Shoaib, S. Alshebeili, AIP Conf. Proc. 1653, 020098 (2015).

[16] M.S. Awan, R. Nebuloni, C. Capsoni, L.C. Horváth, S.S. Muhammad, F. Nadeem, M.S. Khan, E. Leitgeb, Int. J. Satell. Commun. Network. 29, 97 (2011).

[17] E. Ciaramella, Y. Arimoto, G. Contestabile, M. Presi, A. D'Errico, V. Guarino, M. Matsumoto, IEEE Photon. Technol. Lett. 21, 1121 (2009).

[18] M. Siraj, M.I. Memon, M.S. Khan, Acta Phys. Pol. A 129, 826 (2016). 